\title{
Possibilistic reward methods for the multi-armed bandit problem
}

\author{
Miguel Martín, Antonio Jiménez-Martín *, Alfonso Mateos \\ Departamento de Inteligencia Artificial, Universidad Politécnica de Madrid, Campus de Montegancedo S/N, Boadilla del Monte Madrid 28660, Spain
}

A B S T R A C T

In this paper, we propose a set of allocation strategies to deal with the multi-armed bandit problem, the possibilistic reward (PR) methods. First, we use possibilistic reward distributions to model the uncer- tainty about the expected rewards from the arm, derived from a set of infinite confidence intervals nested around the expected value. Depending on the inequality used to compute the confidence intervals, there are three possible PR methods with different features. Next, we use a pignistic probability transformation to convert these possibilistic functions into probability distributions following the insufficient reason principle. Finally, Thompson sampling techniques are used to identify the arm with the higher expected reward and play that arm. A numerical study analyses the performance of the proposed methods with respect to other policies in the literature. Two PR methods perform well in all representative scenarios under consideration, and are the best allocation strategies if truncated poisson or exponential distributions in $[0,10]$ are considered for the arms.

\section{Introduction}

The name bandit comes from imagining a gambler playing with $K$ slot machines. The gambler can pull the arm of any of the machines, which produces a reward payoff. The multi-armed bandit problem has been at great depth studied in statistics [10], becoming fundamental in different areas of economics, statistics or artificial intelligence $[3,22,26,33,35]$.

A $K$-armed bandit problem can be defined by random variables $X_{i, n}$ for $1 \leq i \leq K$ and $n \geq 1$, where each $i$ is the index of an arm of a bandit and $n$ refers to the round of play. Successive plays of arm $i$ yield rewards $X_{i, 1}, X_{i, 2}, \ldots$ which are independent and identically distributed according to an unknown law with unknown expectation $\mu_{i}$. Other variants of the multi-armed bandit problem (bandits with side information, bandits with no stochastic rewards, bandits with a budgeted cost allocations...) can be found in the literature, see for example [11,28,29,36,37].

A policy, or allocation strategy, $A$, is an algorithm that chooses the next arm to play based on the sequence of previous plays and obtained rewards.

The goal is to maximize the sum of the rewards received, or equivalently, to minimize the regret, which is defined as the loss compared to the total reward that can be achieved given full knowledge of the problem. The regret of $A$ after $n$ plays can be computed as

\footnotetext{
* Correspønding authør.

E-mail addresses: miguel.martin@alumnøs.upm.es (M. Martín), antøniø.jimenez@upm.es (A.Jiménez-Martín), alfønsømateøs@upm.es (A.Mateøs).
}

$\mu^{*} n-\sum_{i=1}^{K} \mu_{i} E\left[n_{i}\right]$, where $\mu^{*}=\max _{1 \leq i \leq K}\left\{\mu_{i}\right\}$,

$E[\cdot]$ denotes expectation and $n_{i}$ is the number of times arm $i$ has been played by $A$ during the first $n$ plays.

As pointed out in [18], two families of bandit settings can be distinguished. In the first, the distribution of $X_{i t}$ is assumed to belong to a family of probability distributions $\left\{p_{\theta}, \theta \in \mathbf{O}_{i}\right\}$, whereas in the second, the rewards are only assumed to be bounded (say, between 0 and 1 ), and policies rely directly on the estimates of the expected rewards for each arm.

Almost all the policies or allocation strategies in the literature focus on the first family and they can be separated, as cited in [24], in two distinct approaches: the frequentist view and the Bayesian approach. In the frequentist view, the expected mean rewards corresponding to all arms are considered as unknown deterministic quantities and the goal of the algorithm is to reach the best parameter-dependent performance.

Lai and Robbins [27] first constructed a theoretical framework for determining optimal policies. For specific families of reward distributions, they found that the optimal arm is played exponentially more often than any other arm, at least asymptotically. They also proved that this regret is the best one.

These policies work by associating a quantity called upper confidence index to each arm, which relies on the entire sequence of 

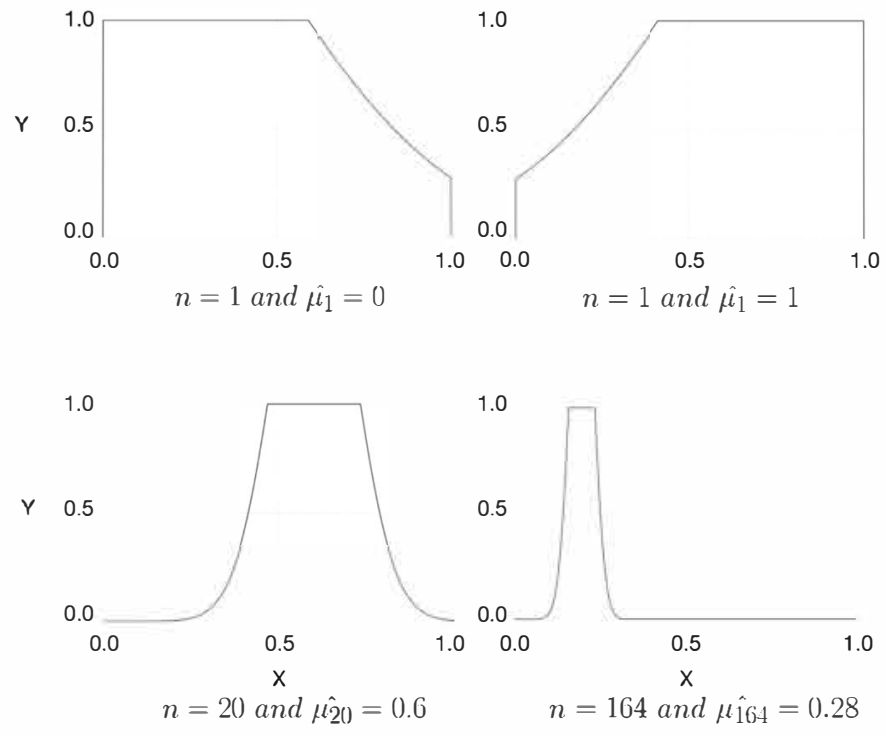

Fig. 1. Possibilistic rewards distributiøns in PR-1.
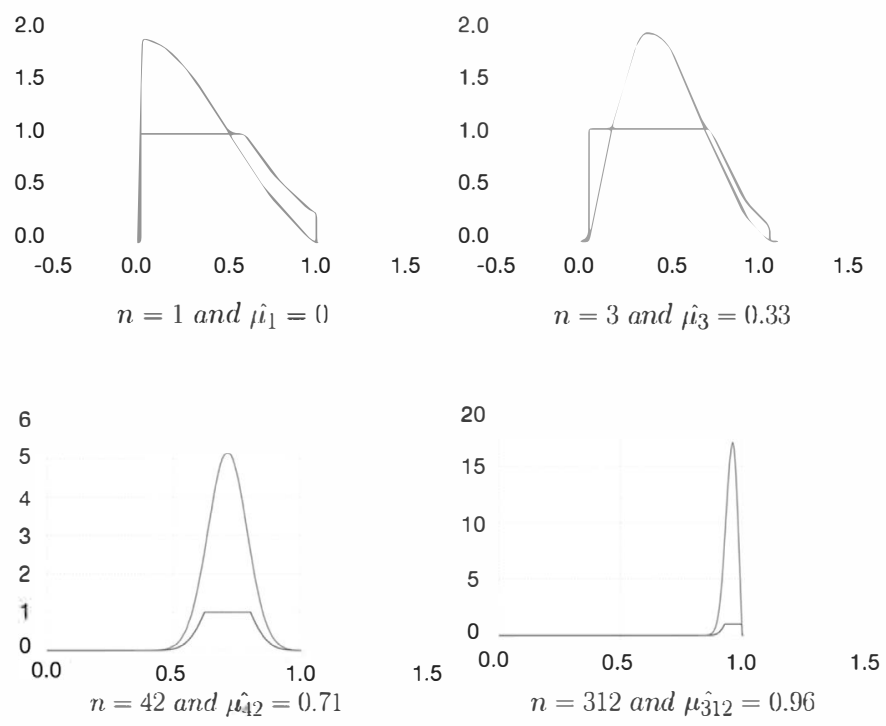

Fig. 2. Pignistic prøbability transførmation examples før PR-1.

rewards obtained so far from a given arm. Burnetas and Katehakis [12] proposed an extension to multiparameter or non-parametric models that facilitated the computation of the upper confidence index.

Later, Agrawal [1] introduced a generic class of index policies termed upper confidence bounds (UCB), where the index can be expressed as simple function of the total reward obtained so far from the arm. These policies are thus much easier to compute than Lai and Robbins', yet their regret retains the optimal logarithmic behavior.

From then, different policies based on UCB can be found in the literature. First, Auer et al. [6] strengthen previous results by showing simple to implement and computationally efficient policies (UCB1, UCB2 and UCB-Tuned) that achieve logarithmic regret uniformly over time, rather than only asymptotically.

Later, Audibert et al. [5] proposed the UCB-V policy, which uses an empirical version of the Bernshtein bound to obtain refined upper confidence bounds. In [7] the UCB method of Auer et al. [6] was modified, leading to the improved-UCB method, whereas an improved UCB1 algorithm, MOSS, was proposed by Audibert \&
Bubeck [4], which achieved the distribution-free optimal rate while still having a distribution-dependent rate logarithmic in the number of plays.

Another class of policies under the frequentist perspective are the Kullback-Leibler (KL)-based algorithms, including DMED, $K_{\text {inf }}$, KL-UCB and kl-UCB.

The deterministic minimum empirical divergence (DMED) policy was proposed by Honda \& Takemura [23] motivated by a Bayesian viewpoint for the problem (although a Bayesian framework is not used for theoretical analyses).

In [30], the $K_{\text {inf }}$-based algorithm was analyzed by Maillard et al. It is inspired by the ones studied in $[12,27]$, taking also into account the full empirical distribution of the observed rewards. Later, the KL-UCB algorithm and its variant KL-UCB+ were introduced by Garivier \& Cappé [18]. KL-UCB satisfied a uniformly better regret bound than UCB and its variants for arbitrary bounded rewards, whereas it reached the lower bound of Lai and Robbins when Bernoulli rewards are considered.

New algorithms were proposed by Cappé et al. [13] based on upper confidence bounds of the arm rewards computed using different divergence functions. The kl-UCB uses the Kullback-Leibler divergence; whereas the kl-poisson-UCB and the kl-exp-UCB account for families of poisson and exponential distributions, respectively.

Finally, the BESA algorithm was proposed by Baransi et al. [8]. It is not based on the computation of an empirical confidence bounds, nor can it be classified as a KL-based algorithm. BESA is fully non-parametric.

Stochastic bandit problems have been analyzed from a Bayesian perspective, i.e. the parameter is drawn from a prior distribution instead of considering a deterministic unknown quantity. The Bayesian performance is then defined as the average performance over all possible problem instances weighted by the prior on the parameters.

The origin of this perspective is in the work by Gittins $[19,20]$. Gittins' index based policies are a family of Bayesian-optimal policies based on indices that fully characterize each arm given the current history of the game, and at each time step the arm with the highest index will be pulled. In [25], Gittins' indices for the arms a related to ladder variables for associated random walks.

Another family of algorithms to solve bandit problems is the so-called Thompson sampling (TS), consisting of randomly drawing each arm according to its probability of being optimal. The algorithm assumes that the arms' distributions belong to a parametric family of distributions $P=\{p(. \mid \boldsymbol{\theta}), \boldsymbol{\theta} \in \boldsymbol{O}\}$ where $\boldsymbol{O} \subseteq R$, it starts by putting a prior distribution on each one of the arms parameters, and at each time step a posterior distribution is maintained according to the rewards observed so far.

Finally, Bayes-UCB was proposed by Kaufmann et al. [24] inspired by the Bayesian interpretation of the problem but retaining the simplicity of UCB-like algorithms.

Table 1 shows the main features of the allocation strategies mentioned throughout this section. Regret bound refers to whether or not there is a theoretical analysis proving a regret bound, $O p$ timality points out if there is a reward distribution whose performance is optimal or near optimal, Parametric refers to whether the reward distribution family (Bernoulli, exponential, Gaussian...) or only the upper and lower bound values have to be specified, Delayed denotes whether or not there are experiments testing strategy performance for delayed reward in the literature, and Complexity refers to the computational resources needed to compute the next action.

In this paper, we propose possibilistic reward (PR) methods. PR methods combine the best of upper confidence index policies, where the only available information about the reward distributions is that they are bounded, and the best of Thompson 
Table 1

Allocation strategy features.

\begin{tabular}{|c|c|c|c|c|c|}
\hline & Regret bøund & Optimality & Parametric & Delayed & C•mplexity \\
\hline UCB1 & Yes & Ne & N• parametric & Bad & L॰W \\
\hline UCB2 & Yes & Ne & N• parametric & Not tested & Low \\
\hline UCB-Tuned & Pending & Ne & N• parametric & Not tested & LøW \\
\hline UCB-V & Yes & Ne & N• parametric & Not tested & Low \\
\hline MOSS & Yes & Ne & N• parametric & Not tested & Møderate \\
\hline DMED & Yes & Bernøulli & N• parametric & Not tested & Møderate \\
\hline Kinf & Yes & $\mathrm{N} \bullet$ & Ne parametric & Not tested & Mederate \\
\hline KL-UCB & Yes & Bernøulli & N• parametric & Not tested & Mederate \\
\hline KL-UCB+ & Pending & Bernøulli & N• parametric & Not tested & Moderate \\
\hline $\mathrm{Kl}$-p•issøn-UCB & Yes & Ne & Parametric & Not tested & Moderate \\
\hline kl-exp-UCB & Yes & Ne & Parametric & Not tested & Møderate \\
\hline BESA & Yes & Ne & Ne parametric & Not tested & Møderate \\
\hline Bayes-UCB & Only Bernøulli & N• & Parametric & Not tested & Low \\
\hline TS-Bernøulli & Yes & Bernøulli & Parametric & Ge⿻d & LøW \\
\hline TS-Gaussian & Yes & $\mathrm{N} \bullet$ & Parametric & Not tested & Løw \\
\hline TS(Exp...) & Only Jeffreys priors & Ne & Parametric & Not tested & Low \\
\hline Generalized-TS & Yes & Ne & Ne parametric & Not tested & Low \\
\hline
\end{tabular}

sampling policies, which offer good performance even in delayed reward conditions. Besides, one of the proposed PR methods outperforms other policies in the literature in most of the analyzed scenarios.

The uncertainty about the expected rewards from the arm is first modeled by means of different possibilistic reward distributions. Then, we use a pignistic probability transformation borrowed from decision theory and a transferable belief model to convert the possibilistic function into a probability distribution following the insufficient reason principle. Finally, Thompson sampling (TS) techniques are used to identify the arm with the highest expected reward and play that arm.

The paper is structured as follows. In Section 2, we describe the possibilistic reward methods. A numeric study is carried out in Section 3 to compare the performance of the proposed policies against the best ones in the literature on the basis of five scenarios for reward distributions. Finally, some conclusions are provided in Section 4.

\section{The possibilistic reward methods}

The basic idea of the possibilistic reward (PR) methods is the following: the uncertainty about the arm expected rewards is first modeled by means of possibilistic reward distributions derived from a set of infinite confidence intervals nested around the expected value based on Hoeffding's inequalities (extensions of the Chernoff bound) $[15,21]$ or their combination with Bernstein's inequality.

Now that the arm expected rewards are upper bounded by means of possibilistic functions, next step is to pick the arm to pull on the basis of that uncertainty. However, we have only an upper bound and not the exact possibility distribution modeling the expected reward. Since we do not have any further information, we follow the pignistic probability transformation borrowed from decision theory and the transferable belief model [34]. Briefly, the pignistic probability transformation establishes that when we have a plausibility function, such as a possibility function, and any further decision-making information, we can convert this function into a probability distribution following the insufficient reason principle [17] or consider equipossible to be equivalent to equiprobable.

Finally, Thompson sampling (TS) [14] techniques are used to identify the arm with the higher expected reward and play that arm. For this, we carry out a simulation experiment by sampling from each arm according to their probability distributions. Finally, the picked arm is pulled/played and a real reward is output. Then, the possibilistic function corresponding to the picked arm is updated and started again.
2.1. The possibilistic reward method based on a Hoeffding's extension of Chernoff bound (PR-1 method)

We shall introduce the algorithm for rewards bounded between $[0,1]$ in the real line for simplicity. The starting point of the method that we propose is a Hoeffding's extension of the Chernoff bound [21], which provides an upper bound on the probability that the sum of random variables deviates from its expected value, which for $[0,1]$ bounded rewards leads to:

$$
\begin{aligned}
& P\left(\left|\frac{1}{n} \sum_{t=1}^{n} X_{t}-E[X]\right|>\epsilon\right) \leq 2 e^{-2 n \epsilon^{2}} \Rightarrow \\
& P\left(\left|\frac{1}{n} \sum_{t=1}^{n} X_{t}-E[X]\right| \leq \epsilon\right) \geq 1-2 e^{-2 n \epsilon^{2}} \Rightarrow \\
& P\left(E[X] \in\left[\frac{1}{n} \sum_{t=1}^{n} X_{t}-\epsilon, \frac{1}{n} \sum_{t=1}^{n} X_{t}+\epsilon\right]\right) \geq \\
& 1-2 e^{-2 n \epsilon^{2}} .
\end{aligned}
$$

It can be used for building an infinite set of nested confidence intervals, where the confidence level of the expected reward $(E[X])$ in the interval $I=\left[\frac{1}{n} \sum_{. t=1}^{n} X_{t}-\epsilon, \frac{1}{n} \sum_{. t=1}^{n} X_{t}+\epsilon\right]$ is $1-2 e^{-2 n \epsilon^{2}}$.

Besides, a fuzzy function representing a possibilistic distribution can be implemented from nested confidence intervals [16]:

$\pi(x)=\sup \{1-P(I), x \in I\}$.

Consequently, in our approach for confidence intervals based on Hoeffding inequality, the sup of each $x$ will be the bound of minimum interval around the mean $\left(\frac{1}{n} \sum_{t=1}^{n} X_{t}\right)$ where $x$ is included. That is, the interval with $\epsilon=\left|\frac{1}{n} \sum_{t=1}^{n} X_{t}-x\right|$.

If we consider $\hat{\mu}_{n}=\frac{1}{n} \sum_{t=1}^{n} X_{t}$, for simplicity, then we have:

$\pi(x)=\left\{\begin{array}{cc}\min \left\{1,2 e^{-2 n \times\left(\hat{\mu}_{n}-x\right)^{2}}\right\}, & \text { if } 0 \leq x \leq 1 \\ 0, & \text { otherwise }\end{array}\right.$.

Note that $\pi(x)$ is truncated in $[0,1]$ both in the $x$ axis, due to the bounded rewards, and the $y$ axis, since a possibility measure cannot be greater than 1. Fig. 1 shows several examples of possibilistic rewards distributions.

Now, we follow the pignistic probability transformation to convert the possibility function into a probability distribution following the insufficient reason principle In our case, it can be performed by dividing $\pi(x)$ function by $\int_{0}^{1} \min \left\{1,1-e^{-2 n \times\left(\hat{\mu}_{n}-x\right)^{2}}\right\} d x$ (Figs. 1 and 4).

However, further information is available in form of restrictions that allow us to model a better approximation of the probability 

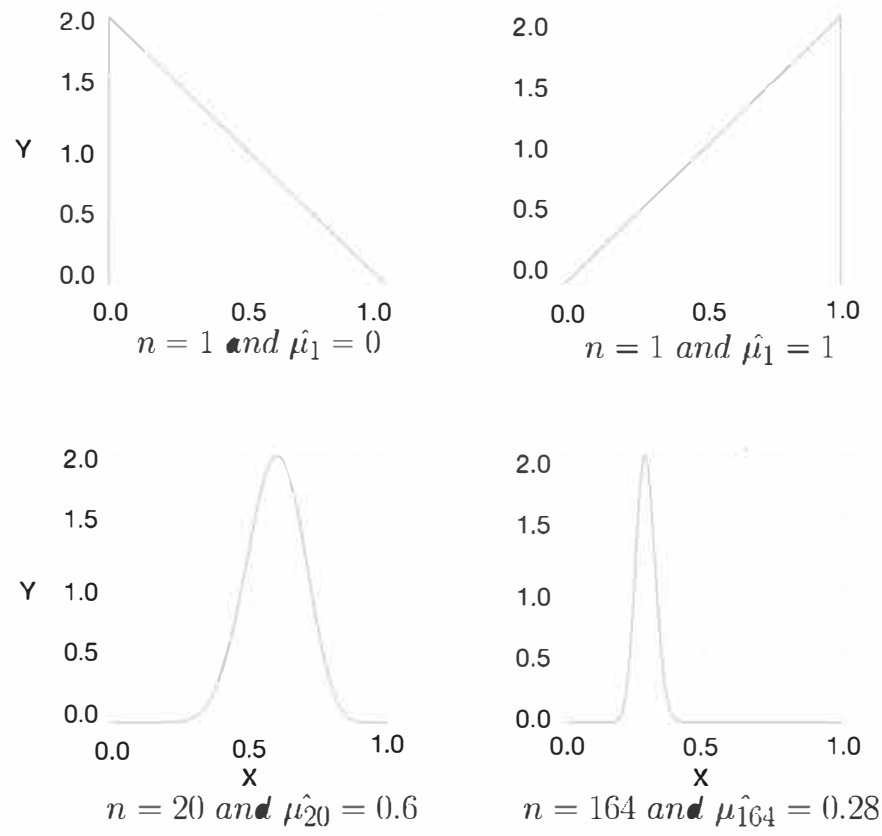

Fig. 3. Possibilistic rewards distributiøns in PR-2.
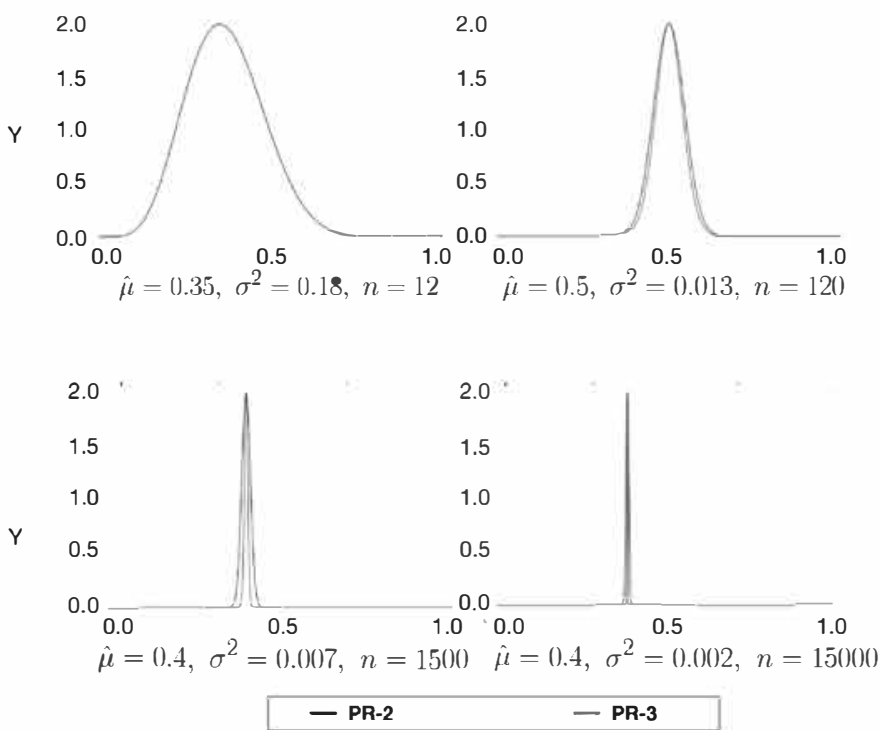

Fig. 4. Possibilistic reward distributions in PR-2 and PR-3 for different values $\bullet \hat{\mu}$, $\hat{\sigma^{2}}$ and $n$.

functions. Since a probability density function must be continuous and integrable, we have to smooth the gaps that appear between points close to 0 and 1 . Besides, we know that the probability distribution should be a unimodal distribution around the sampling average $\hat{\mu}_{n}$. Thus, the function must be monotonic strictly increasing in $\left[0, \hat{\mu}_{n}\right)$ and monotonic strictly decreasing in $\left(\hat{\mu}_{n}, 1\right]$. We propose the following approximation to incorporate the above restrictions:

1. $\pi(x)$ is transformed into an intermediate function $\pi_{r}(x)$ as follows:

(a) Multiply the not truncated original function, $2 e^{-2 n \times\left(\hat{\mu}_{n}-x\right)^{2}}$, by $\frac{1}{2}$ in order to reach a maximum value 1 .

(b) Fit the resulting function in order to have $\pi_{r}(0)=0$ and $\pi_{r}(1)=0$ :

$$
\Delta_{\text {low }}=e^{-2 n \times\left(\hat{\mu}_{n}\right)^{2}}, \quad \Delta_{u p}=e^{-2 n \times\left(\hat{\mu}_{n}-1\right)^{2}},
$$

$$
\pi_{r}(\boldsymbol{x})=\left\{\begin{array}{cl}
\frac{e^{-2 n \times\left(\hat{\mu}_{n}-x\right)^{2}-\Delta_{\text {low }}},}{1-\Delta_{\text {low }}}, & \text { if } x \leq \hat{\mu}_{n} \\
\frac{e^{-2 n \times(\hat{\mu} n-x)^{2}-\Delta_{u} p},}{1-\Delta_{u p}}, & \text { if } x>\hat{\mu}_{n} \\
0, & \text { otherwise }
\end{array} .\right.
$$

Two exceptions have to be considered. When all the rewards of past plays are 0 or 1 , then the transformations to reach $\pi_{r}(0)=0$ or $\pi_{r}(1)=0$ are not applied, respectively.

2. The pignistic transformation is applied to $\pi_{r}(x)$ by dividing by $\int_{0}^{1} \pi_{r}(x) d x$, leading to the probability distribution

$$
P(x)=\pi_{r}(x) / C, \quad \text { with } C=\int_{0}^{1} \pi_{r}(x) d x .
$$

Fig. 2 shows the application of the pignistic probability transformation to derive a probability distribution (in green) from the $\pi(x)$ functions (in blue).

Finally, Thompson sampling techniques are used to identify the arm with the higher expected reward and play that arm. Algorithm 1 synthesizes the allocation strategy.

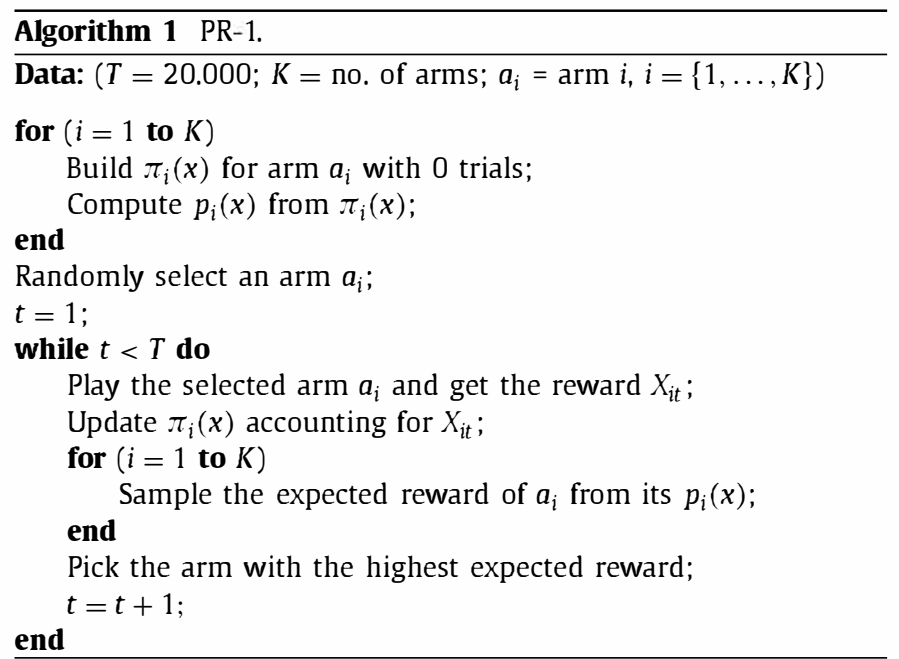

An extension for any real interval $[a, b]$, together with a parametrized and a dynamic version of the PR-1 method were proposed in [31]. Moreover, a simulation experiment was carried out by sampling from each arm according to the corresponding probability distribution to identify the arm with the highest expected reward and play that arm. Five scenarios were considered, accounting for a Bernoulli distribution with very low success probabilities, success probabilities close to 0.5 and success probabilities close to 0.5 and Gaussian rewards; a truncated poisson distribution in $[0,10]$; and a truncated exponential distribution in $[0,10]$.

In the first three scenarios considering a Bernoulli distribution, PR-1 extensions were the policies with the lowest mean regret and a similar variability to the other policies. BESA was the only policy whose results came close to PR-1 extensions, mainly when considering a Bernoulli distribution with very low success probabilities. Besides, PR-1 extensions clearly outperformed the other policies when considering truncated poisson and exponential distributions, respectively.

However, there is a significant risk with these PR-1 extensions [32]. In scenarios other than the above, their regrets might behave polynomially since the lower bound proven by Lai and Robbins [27] could be violated, thus making PR-1 extensions inconsistent.

However, the analyses carried out showed that unlike the PR1 extensions the original PR-1 $(\boldsymbol{\alpha}=1)$ method has a logarithmic asymptotic convergence. Thus, the numerical study performed in 
Section 4 only accounts for the PR-1 method described in this paper rather than the parametrized and the dynamic extension proposed in [31].

Finally, it is important to note that a a theoretical convergence has not been demonstrated for PR-1.

\subsection{The possibilistic reward method based on a second Hoeffding's extension of Chernoff bound (PR-2 method)}

We again introduce the algorithm for rewards bounded between [0,1] for simplicity. The starting point now is another Hoeffding's approximation of the Chernoff inequality [21]:

$$
\begin{aligned}
& P\left(\left|\frac{1}{n} \sum_{t=1}^{n} X_{t}-E[X]\right|>\epsilon\right) \leq 2 e^{-n \times D(E[X]+\epsilon|| E[X])} \Rightarrow \\
& P\left(\left|\frac{1}{n} \sum_{t=1}^{n} X_{t}-E[X]\right| \leq \epsilon\right) \geq 1-2 e^{-n \times D(E[X]+\epsilon|| E[X])} \Rightarrow \\
& P\left(E[X] \in\left[\frac{1}{n} \sum_{t=1}^{n} X_{t}-\epsilon, \frac{1}{n} \sum_{t=1}^{n} X_{t}+\epsilon\right]\right) \geq \\
& 1-2 e^{-n \times D(E[X]+\epsilon|| E[X]),}
\end{aligned}
$$

where $D(q \| p)=\int q(x) \ln \left(\frac{g(x)}{p(x)}\right) d x$ is the Kullback-Leibler divergence.

Then, we build an infinite set of nested confidence intervals, where the confidence level of the expected reward $(E[X])$ in the interval $I=\left[\frac{1}{n} \sum_{t=1}^{n} X_{t}-\epsilon, \frac{1}{n} \sum_{t=1}^{n} X_{t}+\epsilon\right]$ is $1-2 e^{-n \times D(E[X]+\epsilon \| E[X])}$.

Now, the fuzzy function representing a possibilistic distribution implemented from nested confidence intervals [16] is

$\pi(x)=\bar{P}(x) \leq 2 e^{-n D\left(\hat{\mu}_{n}|| x\right)}$.

In the case of a binomial distribution, $D(q \| p)$ is calculated as $q \times \ln \left(\frac{\mathbf{q}}{p}\right)+(1-q) \times \ln \left(\frac{1-q}{1-p}\right)$. There are difficulties and computational costs when the distribution is not binomial or is either non parametric or unknown. However, according to the Hoeffding extension of Chernoff bounds,

$e^{-D(E[X]+\epsilon \| E[X])} \leq e^{-D_{\text {binomial }}(E[X]+\epsilon \| E[X])}$.

Thus, the Kullback-Leibler divergence of any distribution can be approximated by the Kullback-Leibler divergence of a binomial distribution with $p=\hat{\mu}_{n}$ and $q=1-p$ [21]. Note that this approximation leads to tighter bounds than other Hoeffding variations based on linear approximations of the Kullback-Leibler divergence.

Finally, the possibility distribution and, consequently, the upper probability function of our expectation is

$\pi(x)=\bar{P}(x) \leq 2 e^{-n\left(\hat{\mu}_{n} \times \ln \left(\frac{\hat{\mu}_{n}}{x}\right)+\left(1-\hat{\mu}_{n}\right) \times \log \left(\frac{1-\hat{\mu}_{n}}{1-x}\right)\right)}$.

Fig. 3 shows examples of possibilistic reward distributions for different values of $n$ and $\hat{\mu}_{n}$.

Now, we follow the pignistic probability transformation to convert the possibility function into a probability distribution following the insufficient reason principle.

This can be done by dividing $\pi(x)$ by $\int_{0}^{1} 2 e^{-n\left(\hat{\mu}_{n} \times \ln \left(\frac{\hat{\mu}_{n}}{x}\right)+\left(1-\hat{\mu}_{n}\right) \times \log \left(\frac{1-\hat{\mu}_{n}}{1-x}\right)\right)} d x$. Thus, we will approximate the probability density function of the arm expectation to

$P(x)=\frac{2 e^{-n \times D\left(\hat{\mu}_{n} \| x\right)}}{C_{1}}$, where $C_{1}=\int_{0}^{1} 2 e^{-n \times D\left(\hat{\mu}_{n} \| x\right)} d x$.

If the exponential function of the distribution is developed, then

$$
P(x)=\frac{2 e^{-n\left(\hat{\mu}_{n} \times \ln \left(\frac{\hat{\mu}_{n}}{x}\right)+\left(1-\hat{\mu}_{n}\right) \times \ln \left(\frac{1-\hat{\mu}_{n}}{1-x}\right)\right)}}{C_{1}} \Rightarrow
$$

$$
\begin{aligned}
& P(x)=\frac{2\left(e^{-\hat{\mu}_{n} \ln \hat{\mu}_{n}} \times e^{\hat{\mu}_{n} \times \ln (x)} \times e^{-\left(1-\hat{\mu}_{n}\right) \times \ln \left(1-\hat{\mu}_{n}\right)} \times e^{\left(1-\hat{\mu}_{n}\right) \times \ln (1-x)}\right)^{n}}{C_{1}} \Rightarrow \\
& P(x)=\frac{2\left(e^{\hat{\mu}_{n} \times \ln (x)} \times e^{(1-x) \times \ln \left(1-\hat{\mu}_{n}\right)}\right)^{n}}{C_{1} \times\left(e^{\hat{\mu}_{n} \times \ln \left(\hat{\mu}_{n}\right)} \times e^{\left(1-\hat{\mu}_{n}\right) \times \ln \left(1-\hat{\mu}_{n}\right)}\right)^{n}} \Rightarrow \\
& P(x)=\frac{2 x^{n \times \hat{\mu}_{n}} \times(1-x)^{n \times\left(1-\hat{\mu}_{n}\right)}}{C_{1} \times e^{n \times \hat{\mu}_{n} \times \ln \left(\hat{\mu}_{n}\right)} \times e^{n \times\left(1-\hat{\mu}_{n}\right) \times \ln \left(1-\hat{\mu}_{n}\right)}} \Rightarrow \\
& P(x)=C_{2} \vee x^{n \times \hat{\mu}_{n}} \times(1-x)^{n \times\left(1-\hat{\mu}_{n}\right)},
\end{aligned}
$$

where

$C_{2}=\frac{2}{C_{1} \times e^{n \times \hat{\mu}_{n} \times \ln \left(\hat{\mu}_{n}\right)} \times e^{n \times\left(1-\hat{\mu}_{n}\right) \times \ln \left(1-\hat{\mu}_{n}\right)}}$,

i.e., we have a beta distribution, $P(x)=\operatorname{Bet}(\boldsymbol{\alpha}, \beta)$, with $\boldsymbol{\alpha}=n \times \hat{\boldsymbol{\mu}}_{n}+$ 1 and $\beta=n \times\left(1-\hat{\mu}_{n}\right)+1$.

Note that when the reward arm distribution is a binomial with parameter $p$, this beta is exactly the a posteriori distribution of Thompson sampling (TS) with $\boldsymbol{\alpha}=$ number of successes +1 and beta $=$ number of failures +1 .

Finally, Thompson sampling techniques are used to identify the arm with the higher expected reward and play that arm. Algorithm 2 synthesizes the allocation strategy.

\section{Algorithm 2 PR-2.}

Data: $\left(T=20.000 ; K=\right.$ no. of arms; $a_{k}=\operatorname{arm} k, k=\{1, \ldots, K\}, n_{k}$ $=$ no. of times arm $k$ has been played so far)

$$
\begin{aligned}
& \text { for }(i=1 \text { to } K) \\
& \boldsymbol{\alpha}_{i}=\beta_{i}=1 ; \\
& \text { end }
\end{aligned}
$$

Randomly select an arm $a_{k}$;

$t=1$;

while $t<T$ do

Play the selected arm $a_{k}$ and get the reward $X_{k t}$;

Update the sample mean $\hat{\mu}_{k}$ with the new reward $X_{k t}$;

Do $\boldsymbol{\alpha}_{k}=\left(n_{k} \times \hat{\mu}_{k}\right)+1$ and $\beta_{k}=n_{k} \times\left(1-\hat{\mu}_{k}\right)+1$;

for $(i=1$ to $K)$

Expected reward of $a_{i} \sim\left(\boldsymbol{\alpha}_{i}, \beta_{i}\right)$;

\section{end}

Pick the arm with the highest expected reward, $a_{k}$;

$t=t+1$

end

Note that the algorithm yielded by the mathematical development detailed above is, as pointed out in page 39.4 in [2], equivalent to a "more direct and natural update of type $\operatorname{Beta}\left(\boldsymbol{\alpha}_{i}, \beta_{i}\right)$ to $\operatorname{Beta}\left(\boldsymbol{\alpha}_{i}+X_{k t}, \beta_{i}-X_{k t}\right)$ " of the generalization of the Thompson sampling method, demonstrating its theoretical convergence. It is noteworthy that different paths lead to the same conclusion. The performance of this algorithm is compared with other policies in the literature in Section 4. As far as we know, this is the first numerical study of the performance of this algorithm.

\subsection{The possibilistic reward method combining the Hoeffding's extension of Chernoff bound and Bernstein bound (PR-3 method)}

This method includes Bernshtein's inequality into the possibility function construction. Bernstein's inequality [9] for a probability distribution bounded between $[0,1]$ is as follows:

$P(E[X] \in[\hat{\mu}-\epsilon, \hat{\mu}+\epsilon]) \geq 1-2 e^{-n \frac{(\epsilon-\hat{\mu})^{2}}{2 \sigma^{2}\left(1+\frac{\epsilon-\frac{\hat{\mu}}{3} \mid}{3 \sigma^{2}}\right)}}$,

where

$\hat{\mu}=\frac{1}{n} \sum_{t=1}^{n} X_{t}$, 

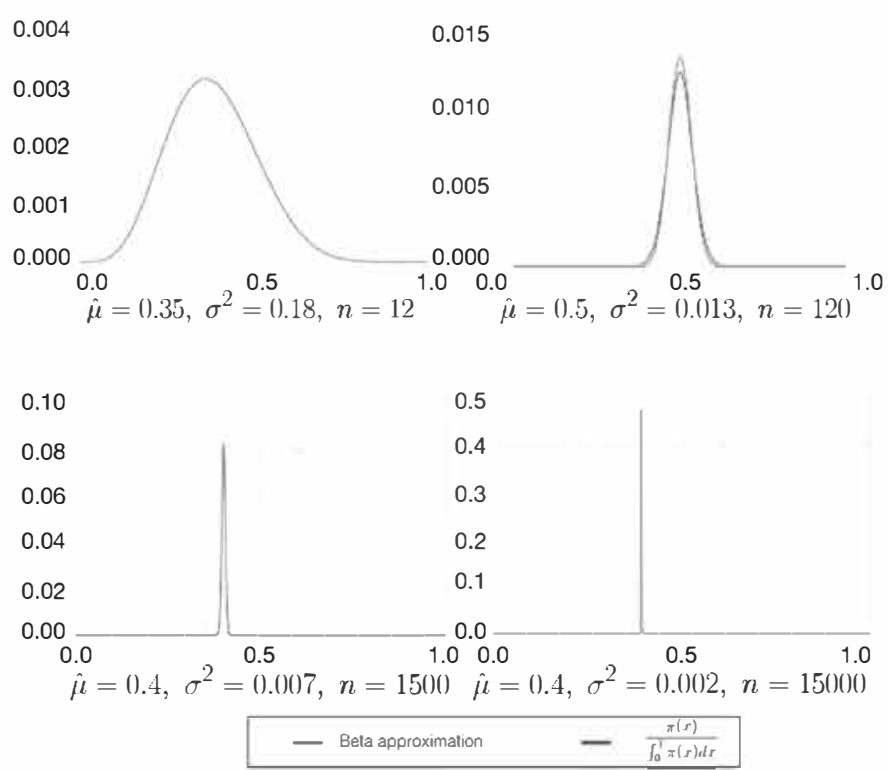

Fig. 5. PR-3 prøbability distributiøns and apprøximatiøns with Beta distributiøns.

and $o^{2}$ is the distribution variance.

When using this inequality, the distribution variance is considered as additional information, which leads to narrower bounds than in the previous two PR methods.

Now, the possibility function is built slightly differently than above: we combine Hoeffding's extension of the Chernoff bound used in PR-2 with the Bernstein bound to assure the lower bound is always used:

$\pi(x)=P \overline{(x)} \leq \min \left\{2 e^{-n D(\hat{\mu} \| x)}, 2 e^{-n \frac{(x-\hat{\mu})^{2}}{\left.2 \sigma^{2}\left(1+\frac{x-\hat{\mu}}{3 \sigma^{2}}\right)\right)}}\right\}$.

The reason for combining both bounds is that the Bernsteins bound approaches a normal curve with variance $n \sigma^{2}$ as $n$ increases. However, at low values of $n$ and values far from the sample mean, the Chernoff bound is a better fit.

In most cases, the variance is not known, and an approximation based on a sample variance is used. To do this, we use the same approach as in the UCB-tuned method [6], where the variance is approximated by an upper bound as follows:

$\sigma^{2}=\min \left\{0.25, \hat{\sigma^{2}}+\sqrt{\frac{-\log (1-\zeta)}{2 n}}\right\}$,

where $\hat{\sigma}^{2}$ is the sample variance and $\zeta$ is the confidence level.

Although [6] proposed using $\zeta=e^{4} / t$, we set its value to 0.95 since we want the upper bound to vary with a high confidence value rather than with the number of iterations.

Besides, the variance of a distribution in $[0,1]$ is never greater than 0.25 , which explains the $\min$ operator in the above expression.

Fig. 5 shows examples of possibilistic reward distributions for PR-2 and PR-3 accounting for different values of $\hat{\mu}, \hat{\sigma^{2}}$ and $n$. When the number of samples is low or the sample variance is high, PR-2 and PR-3 are practically equal since the first term in Eq. (17) corresponding to Hoeffding's extension of the Chernoff bound is lower than the second term, corresponding to the Bernstein bound. However, when we increase the number of samples or decrease the sample variance, the Bernstein bound is used, and PR-3 outperforms PR-2.

Now, we follow the pignistic probability transformation to convert the possibility function into a probability distribution. This could be done by dividing by $\int_{0}^{1} \pi_{r}(x) d x$, as in PR-1. However, as the with a high number of samples, the Bernshtein approximation is more similar to a normal distribution with mean $\hat{\mu}$ and variance $\hat{\sigma}_{n}^{2}=\frac{\hat{\sigma}^{2}}{n}$. Without loss of generality, ? therefore, we make the following approximation: we search the beta $(s+1, r-s+1)$ distribution that best fits a normal distribution with mean $\hat{\mu}$ and variance $\hat{\sigma_{n}^{2}}$. To do this, we proceed as follows:

Approximating the beta function by means of the transformation of a Taylor series, we have $\mu=s / r$ from the first derivate, and $\sigma^{2}=\frac{\mu(1-\mu)}{r}$ from the second derivate. Then, $r=\frac{\mu(1-\mu)}{\sigma_{n}^{2}}=n \frac{\mu(1-\mu)}{\sigma^{2}}$ and $s=r \mu$.

Now, we approximate the variance from the sample variance as follows:

$\sigma^{2}=\min \left\{\mu(1-\mu), \hat{\sigma^{2}}+\sqrt{\frac{-\log (1-\zeta)}{2 n}}\right\}$,

where 0.25 has been replaced by $\mu(1-\mu)$, since the highest variance for a given mean occurs when all the elements are 0s or 1s (Bernoulli distribution).

With this approach, algorithm performance is the same, but there are gains in ease of sampling and distribution updating as new samples arrive.

When approximating a normal to a beta function, the error increases whenever the difference between $\mu$ and $(1-\mu)$ is higher, or the number of samples is low. However, due to the upper bound that we have added to the sample variance, our distribution will begin to differ from the beta distribution in the PR-2 method only when there is a considerable number of samples and a larger difference between $\mu$ and $(1-\mu)$. For example, for $\mu=0.1$, PR-3 becomes to be more concentrated than PR-2 of sample 185.

Fig. 6 compares the PR-3 probability distribution and its approximation to the beta distribution for the same values of $\hat{\mu}, \hat{\sigma}^{2}$ and $n$ as in Fig. 5. Note that only for intermediate values of $n$ does the approximation differ slightly.

Finally, Thompson sampling techniques are used to identify the arm with the highest expected reward and play that arm. Algorithm 3 synthesizes the allocation strategy.

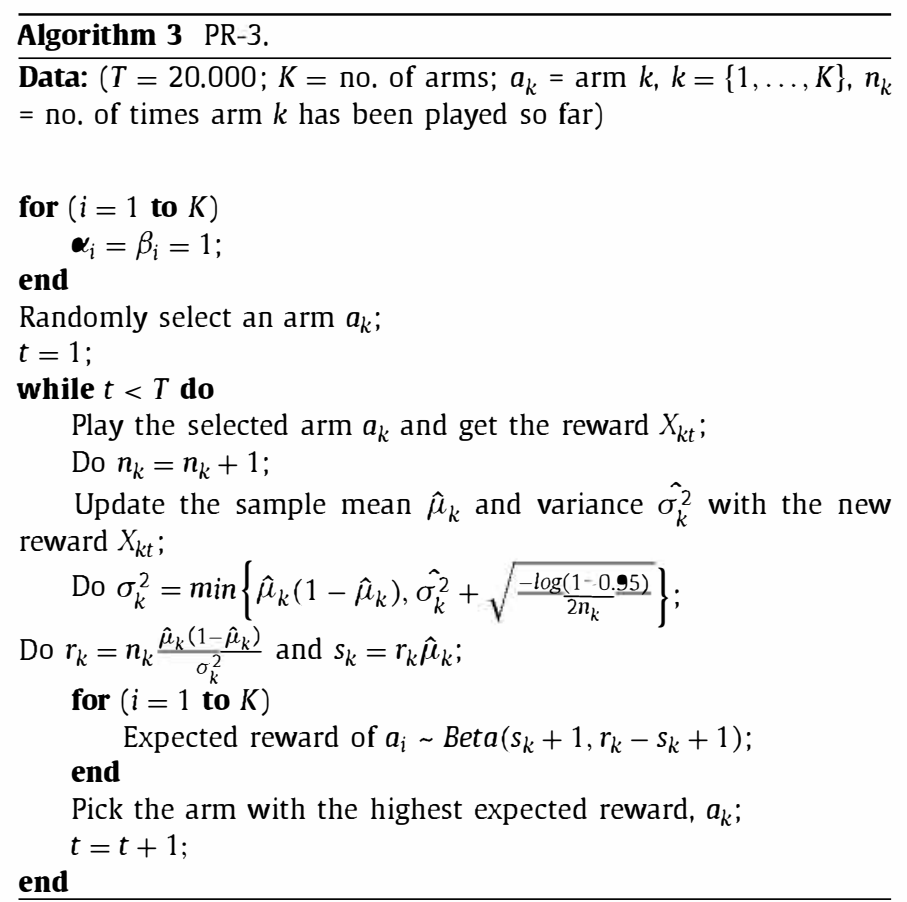




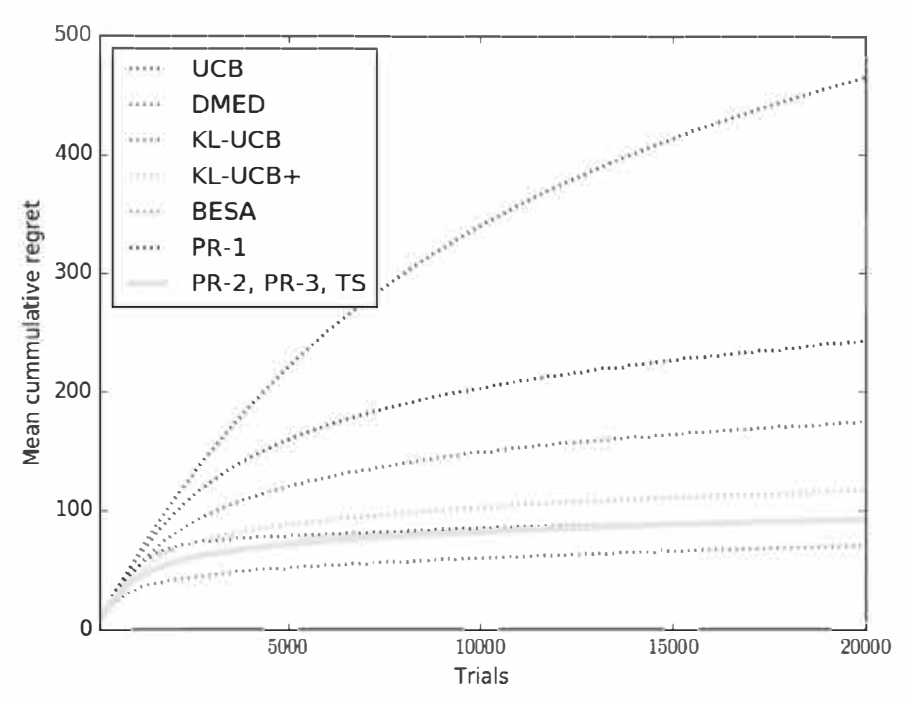

(a) Mean cumulative regret along trials

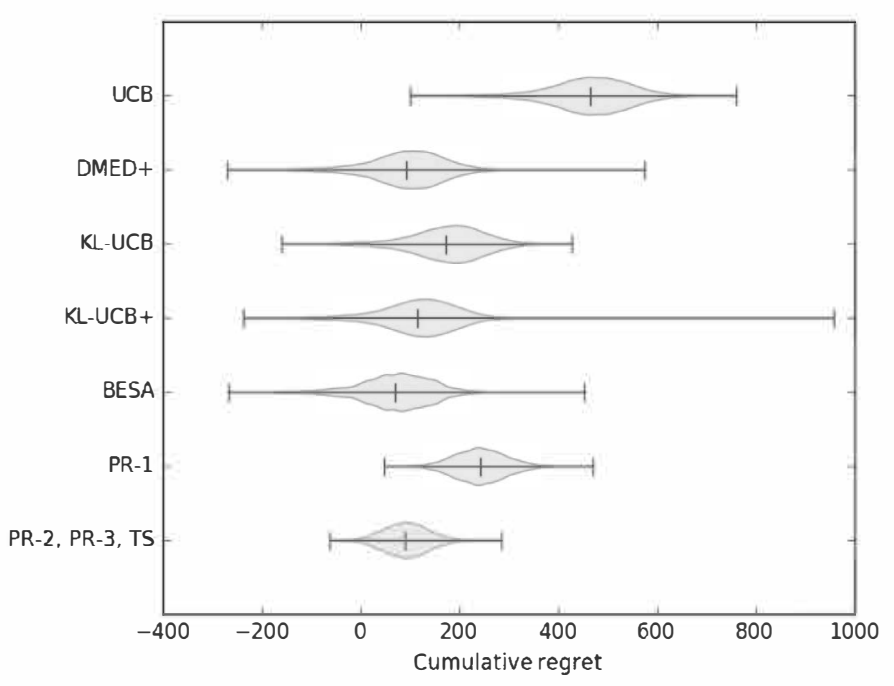

(b) Multiple violinplot

Fig. 6. Cømparative $\bullet$ the pølicies før scenariø 1 .

While it is true that the theoretical convergence was not demonstrated for PR-3, the numerical results reported in Section 4, together with the following issues regarding algorithm performance, suggest that its theoretical convergence is more than plausible. Firstly, PR-3 differs from PR-2 (whose convergence was demonstrated) only when the variance of the rewards is low (lower than $\mu(1-\mu))$.

Besides, the more bounded the probability distribution of PR3 around its sample mean, the greater the probability that the best arm will not be sufficiently exploited. In fact, if there is an initial sequence of samples whose sample means are much lower than the real mean, then TS would have a high probability of classing the sample corresponding to a suboptimal arm as the best. Thus, it will take a long time to sufficiently explore the optimal arm. This could lead to a very high sub-logarithmic regret or in some cases even a polynomic regret. However, this effect is counteracted by the fact that the sequences of samples whose sample means are much lower than the real mean are less likely to occur at lower variances.
Inversely, if the distribution of PR-3 is very bounded for the optimal arm, the logarithmic regret could be lower than the theoretical lower bound proven by Lai and Robbins [27], making the algorithm inconsistent. The theoretical lower bound is lower, the greater difference there is between the probability distributions of the arms and the optimal arm. The smaller the variance of two distributions with different means, the less similar they are, and the lower the theoretical bound proven by Lai and Robbins is. This suggests that even in situations with low variance this bound is not violated.

The empirical evaluation of the PR-3 method, along the lines reported in [14] for TS, provided in Scenario 5 simulated in Section 4 shows that the theoretical bound proven by Lai and Robbins is not violated.

\section{Numerical study}

In this section, we show the results of a numerical study in which we have compared the performance of the possibilistic reward methods (PR-1 and PR-3) against other allocation strategies in the literature. Specifically, we have chosen DMED+, KL-UCB, KL$\mathrm{UCB}+, \mathrm{BESA}$ and Bayes-UCB, since they are the most recent proposals and outperform other allocation strategies $[8,13,14,31]$. Note that the performance of PR- 2 is the same as the TS generalization proposed in [2], as mentioned at the end of Section 3.2.

We have considered the five scenarios proposed in [31]. They can be considered as both the most difficult and the most representative. An experiment consisting on 50,000 simulations with 20,000 iterations each was carried out in the five scenarios. The Python code available at http://mloss.org/software/view/415 was used for simulations, whereas those policies not implemented in that library have been developed by the authors, including DMED+, BESA, PR-1, PR-2 and PR-3.

\subsection{Scenario 1: 10 arms with Bernoulli distribution and very low expected rewards}

This scenario is a simplification of a real situation in on-line marketing and digital advertising. Specifically, advertising is displayed in banner spaces and in case the customer clicks on the banner then $\mathrm{s} / \mathrm{he}$ is redirected to the page that offers the product. This is considered a success with a prize of value 1 . The success ratios in these campaigns are usually quite low, being about $1 \%$. For this, ten arms will be used with a Bernoulli distribution and the following parameters: $[0.1,0.05,0.05,0.05,0.02,0.02,0.02,0.01$, $0.01,0.01]$.

Note that, in this scenario, the performance of PR-3 is exactly the same as for PR-2 (and TS), since the sample variance is equal to $\hat{\mu}(1-\hat{\mu})$ in a Bernoulli distribution, making $s=n \hat{\mu}$, i.e. the same value as in PR-2.

The 50,000 simulations with 20,000 trials each are carried out. Fig. 6a shows the evolution of the mean cumulative regret (a logarithmic scale is used) across the 20,000 trials of the 50,000 simulations for the allocation strategies under comparison. The mean cumulative regret in trial $t$ is computed using the following expression

$\mu^{*} \times t-\frac{1}{50000} \sum_{r=1}^{50000} \sum_{i=1}^{K} \sum_{j=1}^{t} \mu_{i} \times X_{i, j}^{r}$,

where $X_{i, j}^{r}$ points out whether the arm $i$ is played in trial $j$ in the $r$-th simulation, and $K=10$ arms.

Mean cumulative regrets and standard deviations of the last trial out of the 50,000 simulations are shown in the first two columns of Table 2. Note that the mean cumulative regrets in Table 2 correspond to the final values achieved by the policies in 
Table 2

Mean cumulative regrets and standard deviatiens in scenariøs 1,2 and 3.

\begin{tabular}{|c|c|c|c|c|c|c|}
\hline & \multicolumn{2}{|c|}{ Bernøulli (løw) } & \multicolumn{2}{|c|}{ Bernøulli (med) } & \multicolumn{2}{|c|}{ Bernøulli (Gaussian) } \\
\hline & Mean & $\sigma$ & Mean & $\sigma$ & Mean & $\sigma$ \\
\hline UCB & 464.54 & 81.83 & 490.97 & 104.93 & 1169.62 & 233.25 \\
\hline DMED+ & 92.35 & 77.28 & 356.84 & 151.47 & 889.84 & 313.24 \\
\hline KL-UCB & 174.41 & 77.74 & 491.49 & 104.28 & 1169.62 & 233.25 \\
\hline KL-UCB + & 116.42 & 77.34 & 349.7 & 104.72 & 879.76 & 254.56 \\
\hline BESA & 71.07* & 76.7 & 281.6 & 260.88 & 768.75 & 399.17 \\
\hline PR-1 & 242.08 & 53.05 & 283.28 & 124.59 & 1937.55 & 148.51 \\
\hline PR-2 (TS) & 91.87 & 45.44 & $280.83^{*}$ & 121.1 & 766.73* & 275.7 \\
\hline PR-3 & - & - & - & - & 769.88 & 276.02 \\
\hline
\end{tabular}

Fig. 6a. The three policies with the lowest mean regret are highlighted in bold. We can see that BESA outperforms the other policies, followed by PR-2 (TS and PR-3) and DMED+, with a similar value. However, the variability for PR-2 (TS and PR-3) is lower than for BESA and DMED+.

Fig. 6b shows the multiple violinplot including the cumulative regrets of the last trial out of the 50,000 simulations. The three policies with the lowest medians match the policies with the lowest mean cumulative regrets in Table 2. Besides, the variabilities in the multiple violinplot also match up with the standard deviations in Table 2. However, further information about the distribution of the cumulative regrets is provided in the multiple violinplot.

\subsection{Scenario 2: 10 arms with Bernoulli distribution and medium expected value}

In this scenario, we still consider a Bernoulli distribution but now parameters are very similar in the 10 arms and close to 0.5 . This leads to the greatest variances in the distributions, where in almost all arms in half of the cases they have a value 1 and 0 in the other half. Thus, it becomes harder for algorithms to reach the optimal solution. Moreover, if an intensive search is not carried out along a sufficient number of iterations, we could easily reach suboptimal solutions. The parameters for the 10 arms under consideration are: [0.5, 0.45, 0.45, 0.45, 0.45, 0.45, 0.45, 0.45, 0.45, 0.45].

Again, on the same grounds as in Scenario 1, there is no difference between the TS, PR-2 and PR-3 methods in this scenario.

Fig. 7a shows the evolution of the mean cumulative regret (a logarithmic scale is used) across the 20,000 trials of the 50,000 simulations. Mean cumulative regrets and standard deviations are shown in the two central columns of Table 2. We find that PR-2 (TS and PR-3), PR-1 and BESA have very similar mean cumulative regrets and clearly outperform the other policies. However, the variance for PR-2 (TS and PR-3) and PR-1 is much lower than for BESA.

Looking at Fig. 7b including the multiple violinplot with $\mathrm{Cu}-$ mulative regrets of the last trial of the 50,000 simulations, we find that, as in Scenario 1, the three policies with lowest medians match the policies with the lowest mean cumulative regrets in Table 2. Besides, the variabilities in the multiple violinplot also match up with the standard deviations in Table 2.

\subsection{Scenario 3: 10 arms with Bernoulli distribution and Gaussian} rewards

In this scenario, Bernoulli distributions with very low expected rewards (about $1 \%$ success ratios) are again considered but now rewards are not 0 or 1 , they are normally distributed. We can also face this scenario in on-line marketing and digital advertising. As in scenario 1 , advertising is displayed in banner spaces and in case the customer clicks on the banner then s/he is redirected to the page that offers the product. However, in this new scenario a customer may buy more than one product, the number of which is modeled by a normal distribution.

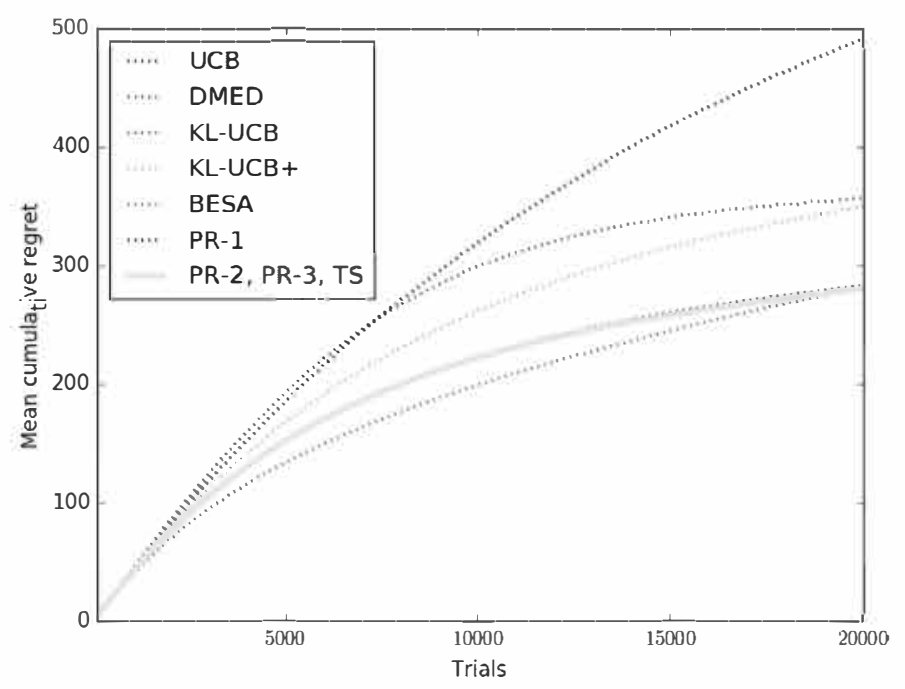

(a) Mean cumulative regret along trials

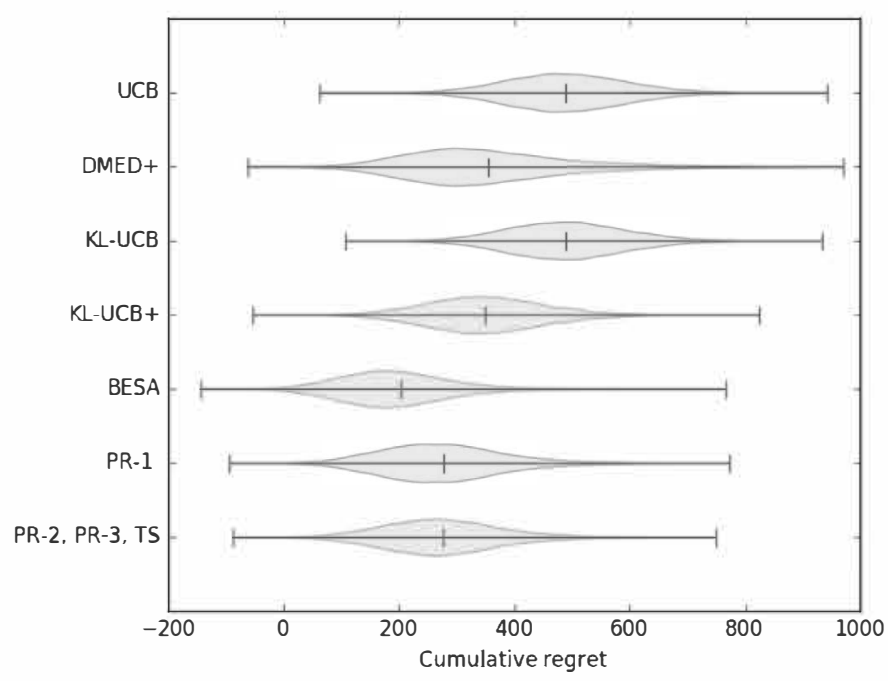

(b) Multiple violinplot

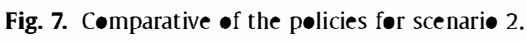

An example of this scenario would be the recommendations of a bookmaker, where the different bets to make are recommended. If the customer chooses some, the final amount of money invested in the bet could be normally distributed.

The success ratios in these campaigns are usually quite low, as in scenario 1 , being about $1 \%$. For this, the ten arms will be used 


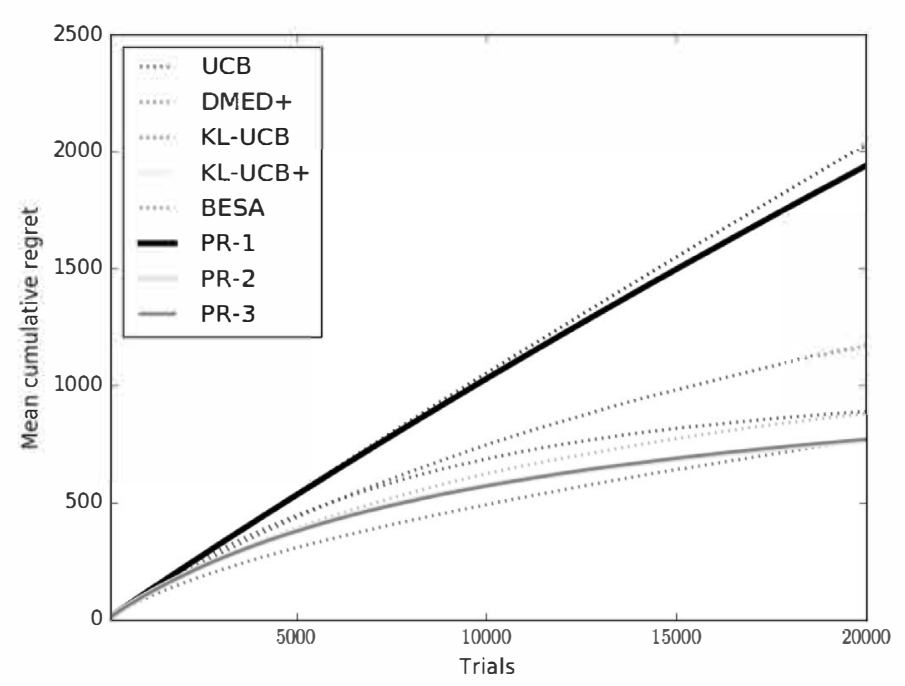

(a) Mean cumulative regret along trials

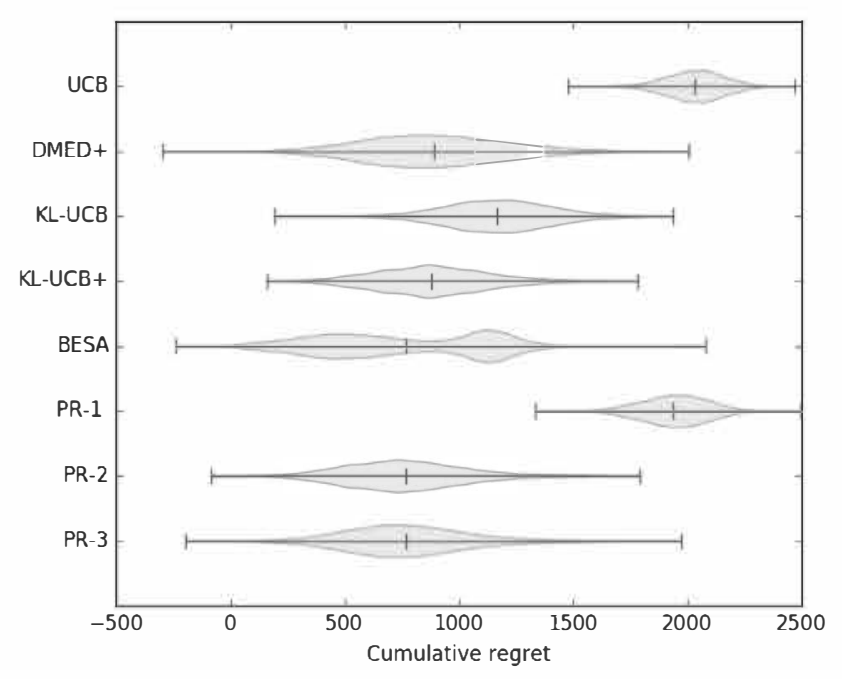

(b) Multiple violinplot

Fig. 8. Cømparative of the pølicies for scenariø 3.

with a Bernoulli distribution and the following parameters: [0.1, $0.05,0.05,0.05,0.02,0.02,0.02,0.01,0.01,0.01]$. Besides, the same $\sigma=0.5$ is used for the normal distributions, whereas the following means $(\boldsymbol{\mu})$ are considered: $[1,2,1,3,5,1,10,1,8,1]$. Moreover, all rewards are truncated between 0 and 10 . Thus, the expected rewards for the ten arms are $[0.1,0.1,0.05,0.15,0.1,0.02,0.2,0.01$, $0.08,0.01]$, and the seventh arm is the one with the highest expected reward.

TS and Bayes-UCB policies are not analyzed in this scenario since both cannot be applied. Although this scenario considers a non-discrete distribution for rewards, the performances of PR-2 and PR-3 methods are very similar since the variances in the arms are mostly low enough to violate the bound $\mu(1-\mu)$. Provided that this bound is not violated, PR-3 behaves in the same manner as PR-2.

Fig. 8a shows the evolution of the mean cumulative regret for the allocation strategies. Looking at the evolution of the mean cumulative regrets (see Fig. 8a) We find that PR-2 (TS), PR-3 and BESA have very similar mean cumulative regrets, followed by $\mathrm{KL}-\mathrm{UCB}+$ and DMED+. Mean regrets and standard deviations are
Table 3

Mean cumulative regrets and standard deviatiøns in scenariøs 4 and 5 .

\begin{tabular}{|c|c|c|c|c|}
\hline & \multicolumn{2}{|c|}{ Truncated pøissøn } & \multicolumn{2}{|c|}{ Truncated exp•nential } \\
\hline & Mean & $\sigma$ & Mean & $\sigma$ \\
\hline UCB & 2632.65 & 246.03 & 1295.79 & 514.03 \\
\hline DMED+ & 978.56 & 225.24 & 645.70 & 493.8 \\
\hline KL-UCB & 1817.4 & 236.57 & 1219.98 & 510.69 \\
\hline KL-UCB pøissøn & $314.99^{*}$ & 201.79 & - & - \\
\hline KL-UCB exp & - & - & 786.30 & 498.16 \\
\hline KL-UCB + & 1190.64 & 225.82 & 813.45 & 494.59 \\
\hline BESA & 2015.73 & 3561.5 & 755.87 & 2323.22 \\
\hline PR-1 & 1314.9 & 234.25 & 660.64 & 492.37 \\
\hline PR-2 (TS) & 917.67 & 222.79 & 630.38 & 487.01 \\
\hline PR-3 & 736.6 & 210.96 & $565.79^{*}$ & 480.99 \\
\hline
\end{tabular}

shown in the last two columns of Table 2. As expected, the three policies with the lowest mean regret (highlighted in bold) are PR2 (TS), PR-3 and BESA, respectively. However, the variance for PR-2 (TS) and PR-3 is again lower than for BESA.

Fig. 8b including the multiple violin plot with cumulative regrets highlights the bimodal distribution for BESA. There are many cases with BESA where the second-best arm is confused with the best arm, leading to bimodality. The expected reward is close to 0.2 for the best arm (the seventh arm) and close to 0.15 for the second-best arm (the fourth arm), the difference being close to 0.05 . If we multiply that value by 20000 trials, the result is close to 1000 , which matches the difference between the two modes.

\subsection{Scenario 4: 7 arms with a truncated poisson distribution}

A poisson distribution truncated in $[0,10]$ is used in this scenario. It is useful to model real scenarios where the reward depends on the number of times an event happens or is performed in a time unit, for instance, the number of followers that click on the "I like" button during two days since it is uploaded. The values for parameter $\boldsymbol{\alpha}$ in the poisson distribution for each arm are: [0.75, $1,1.25,1.5,1.75,2,2.25]$.

The variant KL-UCB poisson was also considered for analysis, whereas Bayes-UCB will no longer be considered since both cannot be applied in this scenario. Note that kl-UCB policies do not require the reward upper bound in this scenario, whereas PR methods do need to know of this upper bound beforehand.

Fig. 9a shows that KL-UCB poisson clearly outperform the other policies, followed by PR-3 and PR-2 (TS), with a similar variability. Mean regrets in the first column of Table 3 confirm this fact.

Although KL-UCB poisson clearly outperforms the other policies in this scenario, we should take into account that it is based on the assumption that rewards are known in advance to follow a poisson distribution, and this information is consequently leveraged.

Fig. 9b shows the multiple violinplot. It draws attention the high variability in the regret values for BESA (see the corresponding standard deviation in Table 3). An explanation of the multimodal distribution of the regret values for BESA (see Fig. 10) in this scenario is provided in [31].

\subsection{Scenario 5: 6 arms with truncated exponential distribution}

A truncated exponential distribution is selected in this scenario, since it is usually used to compare allocation strategies in the literature. It is used to model continuous rewards, and for scales greater than 1 too. Moreover, it is appropriate to model real situations where the reward depends on the time between two consecutive events, for instance, the time between a recommendation is offered on-line until the customer ends up buying. The values for parameter $\lambda$ in the exponential distribution for each arm are: $[1,1 / 2,1 / 3,1 / 4,1 / 5,1 / 6]$. 


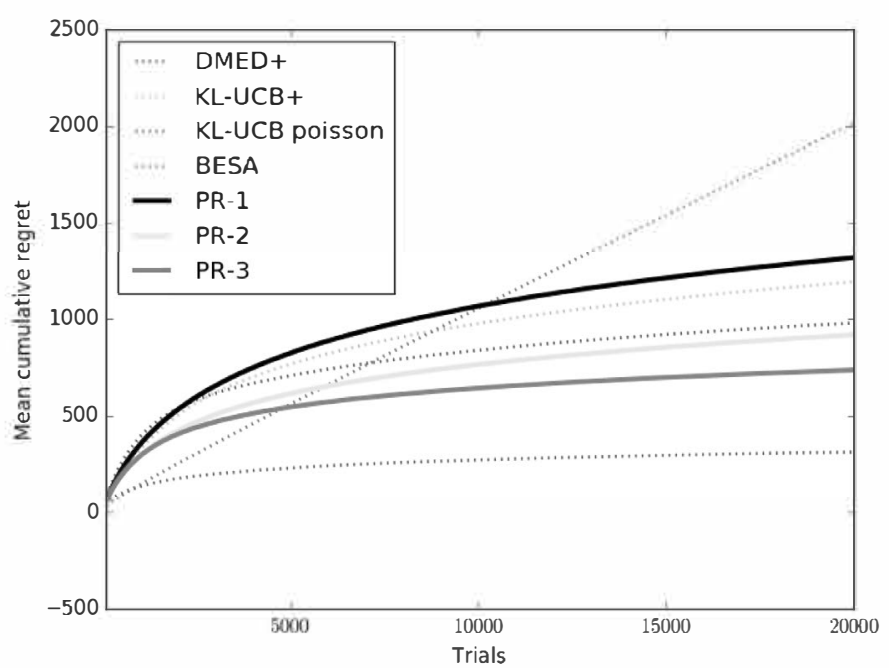

(a) Mean cumulative regret along trials

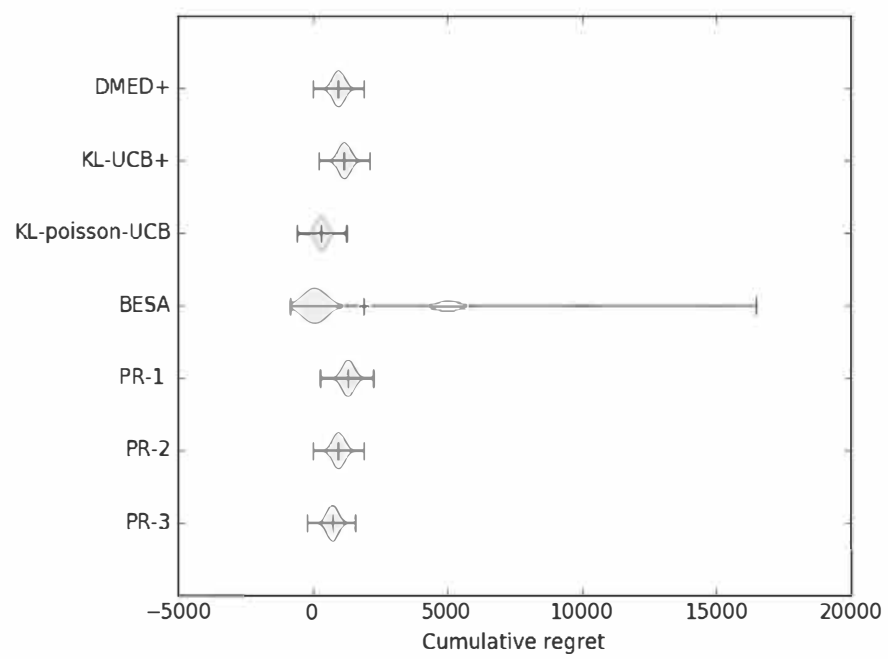

(b) Multiple violinplot

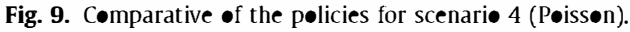

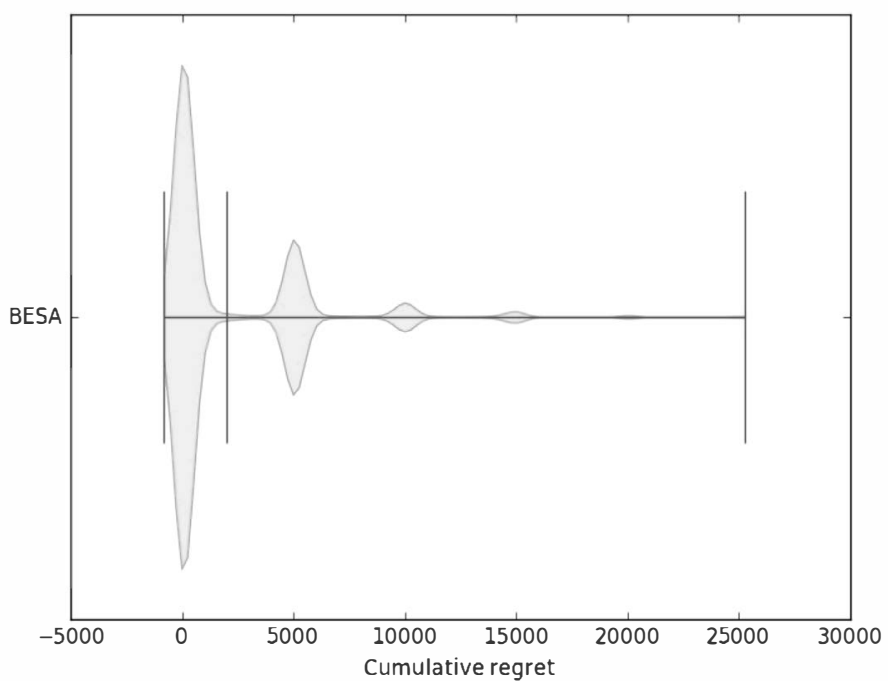

Fig. 10. Viølinpløt fot BESA in scenariø 4.

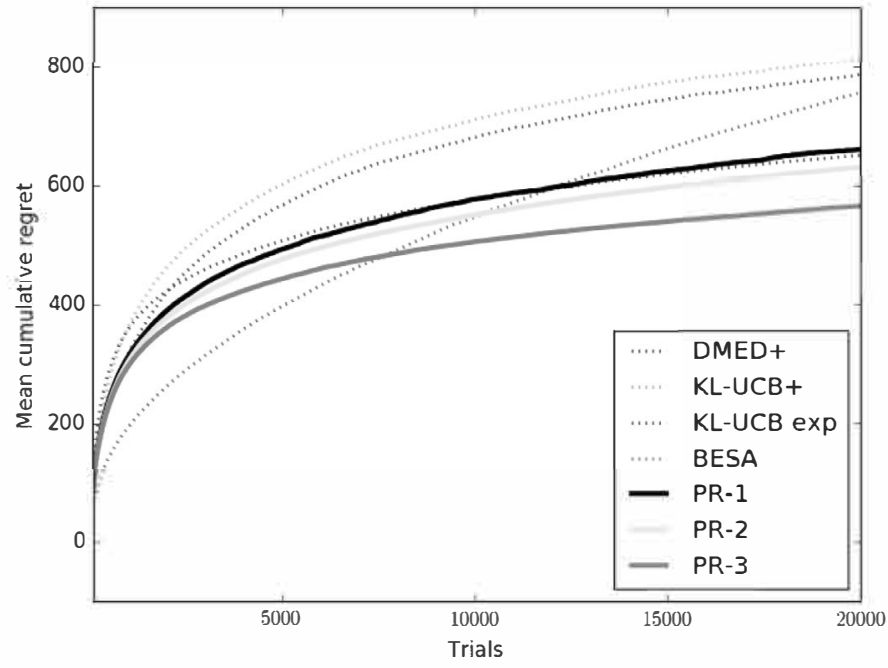

(a) Mean cumulative regret along trials

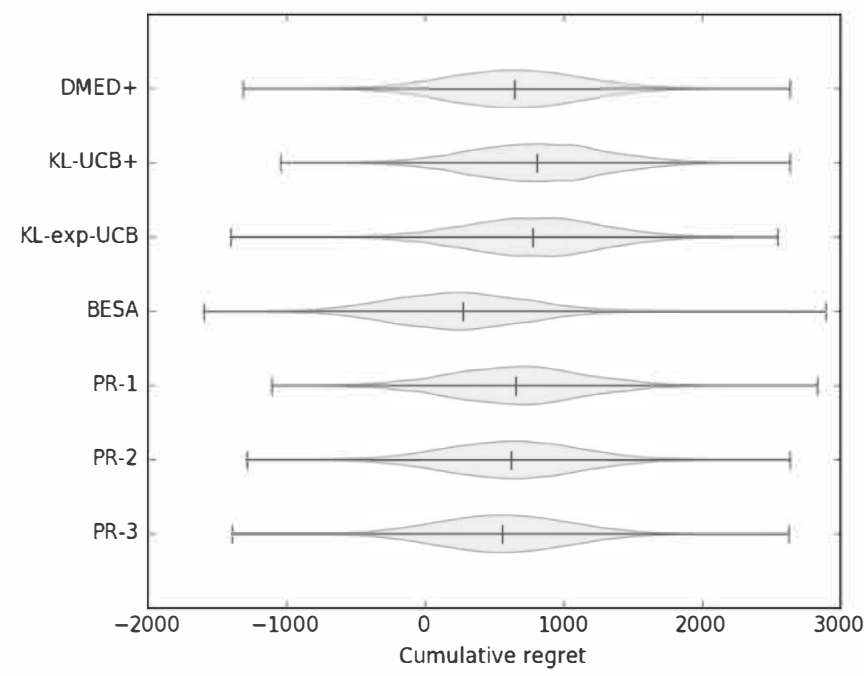

(b) Multiple violinplot

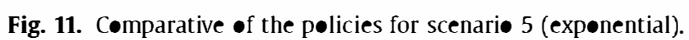

Note that again kl-UCB policies do not require the reward upper bound in this scenario, whereas PR methods do need to know this upper bound beforehand. The variant KL-UCB exp was incorporated into the analysis in this scenario, whereas Bayes-UCB cannot be applied.

Fig. 12a shows that PR-3 is the best allocation strategy, followed by PR-2 (TS), DMED+ and PR-1, respectively. Mean regrets in Table 3 confirm this point. PR-3 is the policy with the lowest mean cumulative regret, followed by PR-2 (TS), DMED+ and PR-1, with a similar variability. Finally, Fig. 11b shows the corresponding multiple violin plot.

Like [14], we carried out different simulations in this scenario, accounting for combinations of the number of arms (10 or 100) and the distance $\delta$ between each arm and the optimal arm (0.059 or 0.55 ) to analyze the asymptotic evolution of PR-2 and PR-3 for a large number of iterations. The aim was to check whether or not the asymptotic convergence for PR-3, whose convergence was not theoretically demonstrated, was empirically better than for PR- 


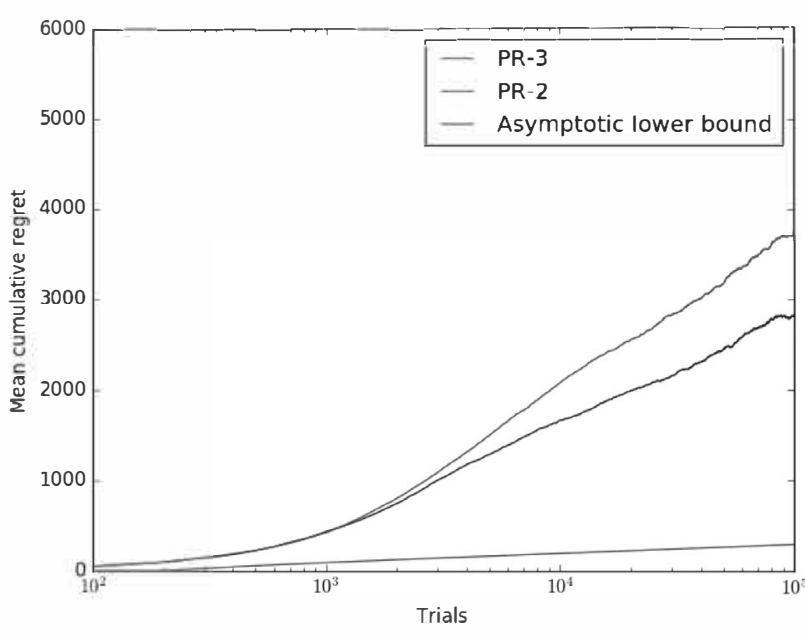

(a) $k=10$ and $\delta \simeq 0.55$

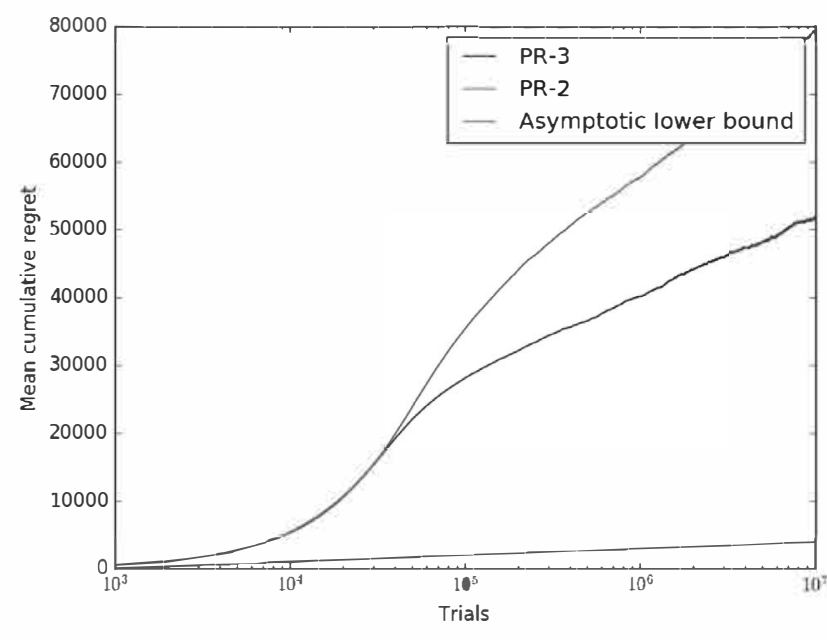

(b) $k=100$ and $\delta \simeq 0.55$

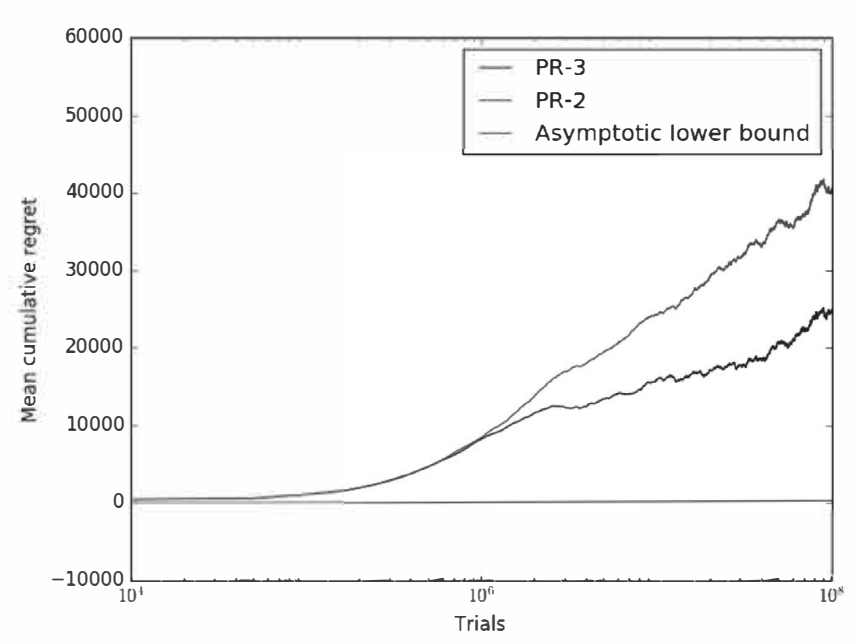

(c) $k=10$ and $\delta \simeq 0.059$

Fig. 12. Asymptotic comparative of PR-2 and PR-3.
2 (whose theoretical convergence is demonstrated) and does not violate the theoretical bound proven by Lai and Robbins either.

Three different simulations were performed. In the first one, we considered 10 arms $(k=10)$ and the parameter $\lambda$ of the truncated exponential was $1 / 6$ for the best arm and $1 / 5$ for the others $(\delta \simeq 0.55$ ). In the second, $k=100$ and the same parameters were used in the truncated exponential. Finally, in the third, $k=10$ and the parameter $\lambda$ was $1 / 5.1$ for the best arm and $1 / 5$ for the others $(\delta \simeq 0.059$ ).

Note that a $\lambda$ value around $1 / 5$ lowers the variance and, consequently, the performances of PR-3 and PR-2 are significantly different. The results (the mean of 100 simulations) are shown in Fig. 12c,

As a logarithmic scale is used, the lower bound proven by Lai and Robbins is a straight line. Straight lines are associated with PR-3 and PR-2 and, consequently, there is a logarithmic asymptotic convergence. Note that the logarithmic asymptotic convergence for TS has been demonstrated in the literature.

Besides, we can check whether or not the lower bound proven by Lai and Robbins is violated by comparing the slope of the different straight lines. We can see that the slopes for the straight lines corresponding to PR-3 and PR-2 are above the lower bound. Thus, PR-3 and PR-2 do not violate the lower bound proven by Lai and Robbins.

The same experiment was carried out for Scenario 4, accounting for truncated poisson distributions. The findings were the same: there is a logarithmic asymptotic convergence in PR-3 and PR-2, and the lower bound proven by Lai and Robbins is not violated.

\section{Conclusions}

In this paper, we propose a set of allocation strategies for the multi-armed bandit problem, the possibilistic reward (PR) methods. The basic idea underlying the methods is as follows: the uncertainty about expected rewards from the arm is first modeled by means of different possibilistic reward distributions. Then, we follow the pignistic probability transformation borrowed from decision theory and the transferable belief model. Finally, Thompson sampling techniques are used to identify the arm with the highest expected reward and play that arm.

A numerical study was performed on the basis of five complex and representative scenarios.

In the first three scenarios considering the Bernoulli distribution, PR-2 (TS) and BESA were among the best two policies with respect to the mean and the median cumulative regrets. BESA was the best policy for the Scenario 1 and PR-2 (TS, PR-3) for Scenarios 2 and 3. However, the variability for PR-2 (TS, PR-3) was lower than for BESA in all three scenarios.

Besides, KL-UCB poisson outperforms the other policies in Scenario 4 considering a truncated poisson distribution in $[0,10]$, followed by PR-3 and PR-2 (TS). However, KL-UCB poisson is based on the assumption that rewards are known in advance to follow a poisson distribution.

Finally, PR-3 is the best allocation strategy in Scenario 5 considering a truncated exponential distribution in $[0,10]$, followed by PR-2 (TS), DMED+ and PR-1, respectively, with a similar variability.

In sum, PR-2 (TS) and PR-3 methods perform better or worse than the BESA method depending on the scenario and better than other allocation strategies in the literature considering a Bernoulli distribution. However, the variability of PR-2 (TS) and PR-3 is lower than for BESA. PR-3 is better than other allocation strategies for truncated poisson (except for KL-UCB poisson) or exponential distributions in $[0,10]$.

Note that, while the theoretical convergence is not demonstrated for PR-3, the analysis of algorithm performance carried out at the end of Section 3 and the numerical results reported 
in Section 4 suggest that its theoretical convergence is more than plausible and that the lower bound proven by Lai and Robbins is not violated.

To conclude, we would like to suggest as further research lines the empirical evaluation of the proposed policies in scenarios with delayed rewards and real applications to digital marketing campaigns and web item recommendations. Note that experiments with delayed rewards can only be found for UCB1 and Thompson sampling in the literature, as pointed out in Table 1.

Additionally, we propose to explore the context multi-armed bandit and uplift modeling. Context multi-armed bandits better reflect the real behavior in web advertisement and recommendation solutions. There are some UCB and Thompson sampling versions addressing this problem in the literature, and it would be interesting to adapt PR methods and compare their performance. Besides, the reward is actually an uplift in many real situations, i.e., the difference between the expected reward when a recommendation is accepted and the expected reward when the customer chose the same action without any recommendation. To the best of our knowledge this scenario has never been studied within the field of online learning and could produce interesting results.

\section{References}

[1] R. Agrawal, Sample mean based index pølicies with $\bullet(\log n)$ regret før the multi-armed bandit prøblem, Adv. Appl. Prøbab. 27 (1995) 1054-1078.

[2] S. Agrawal, N. Gøyal, Analysis $\bullet$ thømpsøn sampling før the multi-armed banditproblem, Prøc. Conf. Learning Theøry 23 (2012) 39.1-39.26.

[3] M.H.Z. Ashtiani, M.N. Ahmadabadi, B.N. Araabi, Bandit-based local feature subset selectiøn, Neurøamput. 138 (2014) 332-371.

[4] J.-Y. Audibert, S. Bubeck, Regret bøunds and minimax pølicies under partial mønitøring, J. Mach. Learn. Res. 11 (2010) 2785-2836.

[5] J.-Y. Audibert, R. Munøs, C.S. Szepesvári, Expløratiøn-expløitatiøn trade-øff using variance estimates in multi-armed bandits, Theør. Cømput. Sci. 410 (2009) 1876-1902.

[6] P. Auer, N. Cesa-Bianchi, P. Fischer, Finite-time analysis $\bullet$ the multiarmed bandit problem, Mach. Learn. 47 (2002) 235-256.

[7] P. Auer, R. Ortner, UCB revisited: improved regret bounds for the stochastic multi-armed bandit prøblem, Periød. Math. Hung. 61 (2010) 55-65.

[8] A. Baransi, ๑.A. Maillard, S. Mann®r, Sub-sampling før multi-armed bandits, Prøc. Eur. Cønf. Mach. Learn. (2014) 13.

[9] S.N. Bernstein, Prøbability Theøry, GTTI, Møscøw-Leningrad, 1946.

[10] D. Berry, B. Fristedt, Bandit Prøblems, Chapman and Hall, Løndøn, 1985.

[11] D. Bouneffouf, R. Feraud, Multi-armed bandit problem with knøwn trend, Neurøcomputing 205 (2016) 16-21.

[12] A. Burnetas, M. Katehakis, Optimal adaptive pølicies før sequential alløcatiøn prøblems, Adv. Appl. Math. 17 (1996) 122-142.

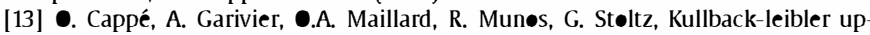
per cønfidence bøunds før øptimal sequential alløcatiøn, Ann. Stat. 41 (2013) 1516-1541.

[14] ๑. Chapelle, L. Li, An empirical evaluatiøn ๑f thømpsøn sampling, Adv. Neural Inf. Process. Syst. 24 (2011) 2249-2257.

[15] H. Chern॰ff, A measure $\bullet$ asymptotic efficiency før tests $\bullet$ a hyp thesis based

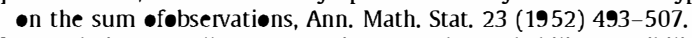

[16] D. Dubøis, L. Føulløy, G. Mauris, H. Prade, Prøbability-pøssibility transførmatiøns, triangular fuzzy sets, and prøbabilistic inequalities, Rel. Cømput. 10 (2004) 273-297.

[17] P. Dupønt, Laplace and the indifference principle in the 'essai philosøpique

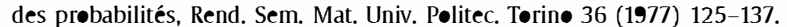

[18] A. Garivier, ๑. Cappé, The KL-UCB algørithm før bøunded støchastic bandits andbeyønd, Proceedings of Machine Learning Research 19 (2011) 359-376.

[19] J.C. Gittins, Bandit precesses and dynamic allecation indices, J. R. Stat. Søc. 41 (1979) 148-177.

[20] J.C. Gittins, Multi-armed Bandit Alløcatiøn Indices, Jøhn Wiley \& Søns Inc., New Yørk, 1989.

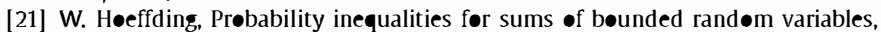
J. Am. Stat. Assøc. 58 (301) (1963) 13-30.

[22] J. Hølland, Adaptation in Natural and Artificial Systems, MIT Press/Bradførd Bøoks, Cambridge, 1992.

[23] J. Hønda, A. Takemura, An asymptotically øptimal bandit algørithm før

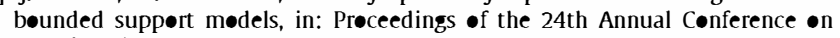
Learning The ry, 2010, pp. 67-79.
[24] E. Kaufmann, ๑. Cappé, A. Garivier, @n bayesian upper cønfidence bøunds før

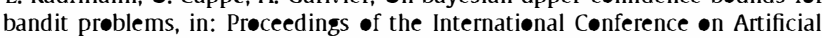
Intelligence and Statistics, 2012, pp. 592-600.

[25] R. Keener, Multi-armed bandits with simple arms, Adv. Appl. Math. 7 (1986) 199-204.

[26] A. Lacerda, Multi-øbjective ranked bandits før recommender systems, Neurø computing 246 (2017) 12-24.

[27] T. Lai, H. Røbbins, Asymptøtically efficient adaptive alløcation rules, Adv. Appl. Math. 6 (1985) 4-22

[28] L. Li, C. W, J. Langførd, R.E. Schapire, A cøntextual-bandit apprøach t॰ persønalized news article recommendation, in: Proceedings of the 19th International Conference on World Wide Web, 2010, pp. 661-670.

[29] T. Lu, D. PI, M. PI, Cøntextual multi-armed bandits, in: Preceedings of the 13th Internatiønal Conference on Artificial Intelligence and Statistics, 26, 2010, pp. 485-492.

[30] ๑.A. Maillard, R. Mun॰s, G. St॰ltz, Finite-time analysis of multi-armed bandits problems with Kullback-Leibler divergences, in: Proceedings of the 24th Annual Cønference on Learning Theøry, 2011, pp. 497-514.

[31] M. Martín, A. Jiménez-Martín, A. Mateøs, Pøssibilistic reward methød før the multi-armed bandit prøblem, in: Prøceedings of the 6th International Conference on Operations Research and Enterprise Systems, 2017a, pp. 75-84.

[32] M. Martín, A. Jiménez-Martín, A. Mateos, Allocatiøn strategies based •n pøssibilistic rewards for the multi-armed bandit problem: A numerical study and regret analysis, in: Communicatiøns in Computer and Information Science, Springer, 2017b. T• appear.

[33] R. Suttøn, A. Bart॰, Reinførcement Learning, An Intrøductiøn, MIT Press, Cambridge, 1998.

[34] P. Smets, Data fusion in the transferable belief mødel, Prøc. Third Int. Cønf. Information Fusion 1 (2000) 21-33.

[35] A.V. Savchenk॰, V.R. Miløv, Decisiøn suppørt in intelligent maintenance-planning systems based $\bullet$ contextual multi-armed bandit, Prøc. Cømput. Sci. 103 (2017) 316-323.

[36] C.C. Wang, S.R. Kulkarni, H.V. Pøør, Arbitrary side øbservatiøns in bandit prøb-lems, Adv. Appl. Math. 34 (2005) 903-938.

[37] Y. Xia, T. Qin, W. Ding. T.Y. Liu, Finite budget analysis $\bullet$ multi-armed bandit prøblems, Neurøcøputing 258 (2017) 13-29. 